\title{
Digital Transformation of Radio Broadcasting: An Exploratory Analysis of Challenges and Solutions for New Digital Radio Services
}

\author{
Stefan Hirschmeier \\ University of Cologne \\ hirschmeier@wim.uni-koeln.de
}

\author{
Roman Tilly \\ University of Cologne \\ tilly@wim.uni-koeln.de
}

\author{
Vanessa Beule \\ University of Cologne \\ beule@wim.uni-koeln.de
}

\begin{abstract}
Like other media industries before, radio broadcasting is increasingly facing competition from new media platforms and changing consumer expectations. Many broadcasters are experimenting with possible solutions and are changing their production processes. While this is necessary, research is needed to capture the whole phenomenon of digital transformation of radio broadcasting. We conducted exploratory qualitative content analysis on talks of radio practitioner to identify current challenges, possible solutions, and specific aesthetics that shape current and future radio experience. We conceptualize the case of digital transformation of radio from the perspective of service-dominant logic and digital service innovation and discuss relevant areas of service innovation. We thus offer orientation for practitioners and contribute to a rather new, yet fruitful area of research for the information systems discipline.
\end{abstract}

\section{Introduction}

Like other industries, radio broadcasting is affected by a digital transformation and is currently undergoing major changes. Whereas music and film industry are already advanced in the process of digital transformation due to pent-up pressure to rearrange their business models, radio broadcasters have experienced less pressure so far. However, they have recently been trying to leap up by providing their content on mobile devices as a first step towards digital transformation. Similar to significant advances in music and film industry, the radio industry also finds itself under increasing pressure to innovate.

Broadcasting agencies are facing strong competition for listeners' attention by new music streaming services and new multimedia platforms. Spotify had 159 million [1] active users in 2017 and 70 million paid subscribers in January 2018 and is the market leader for music streaming services in many countries [2]. Since listeners' time and attention are limited resources, increasing popularity and market share for music and video streaming services negatively affect consumption of radio by listeners.

Broadcasters already react by inventing new channels to distribute their content, by partnering with other broadcasters, and by building up own software departments. Joint projects like radioplayer.uk or radioplayer.de of both public and private radio broadcasters demonstrate that the industry breaks up old political frontiers to open up for change. Nevertheless, only few radio broadcasters, such as US-based National Public Radio, are trying to take full advantage of digitalization and have launched fundamentally new services that combine appealing interfaces with cutting edge technologies like recommender systems.

Many broadcasters are experimenting how to innovate their offerings. While this is necessary to build up experience and knowledge regarding new digital technologies and their possibilities, single agencies may run the risk of missing the "big picture" of digitally transformed radio and may fall short of innovating the industry as a whole. Hence, we ask: What do radio broadcasters do to digitally innovate their offerings? And which theoretical lens can provide useful guidance in further transforming radio broadcasters' offerings?

Although digital transformation, changing business models, value propositions of new radio services and their influence on listeners could present fruitful avenues for research objects for the information systems (IS) discipline, there has been surprisingly little research on challenges and changes resulting from the digital transformation of radio broadcasting. While researchers from various disciplines may feel addressed to provide answers, the IS discipline in particular can provide valuable input to Radio Broadcasting Agencies (RBAs) because digital technologies are and will continue to be pivotal for innovations in radio broadcasting, and 
because many new competitors of broadcasters are genuinely digital companies. Hence, our research goal and contribution are twofold. First, given the lack of research on digital transformation in the radio broadcasting industry, we develop a phenomenological account-based on expert practitioners' assessments - of current challenges for both public and private radio broadcasting agencies. Second, we conceptualize the case of current challenges and changes in the radio broadcasting industry from a service-dominant logic perspective as service innovation [3], [4]. Thus, we can identify important areas for action for RBAs and provide suggestions for innovations based on digital technologies.

\section{Literature Review}

Digital transformation is a phenomenon that has become increasingly important over the last decades [5]-[7]. While the phenomenon seems to be farreaching and complex, and its boundaries unclear [8], we see as a minimal definition that digital transformation is an umbrella term that captures those changes in economy and society that are driven by the pervasion of ever more aspects of every-day life by digital technologies [9]. With respect to enterprises, it is "the use of technology to radically improve performance or reach of enterprises" [7]. Digital technologies include, for example, smart objects, the internet of things, business intelligence, 3D printing, and social media. They are already changing business strategies [10], products and services offered to consumers [11], social relations of all kinds [12] and many more. Some changes can be directly attributed to single technologies or digitized elements and can thus be seen as rather local changes. However, one can also observe more complex and far-reaching changes that depend on a combination of multiple digital technologies and their diffusion. That is, as more and more elements of businesses, economies, and societies become digitized, new systemic possibilities emerge that cannot be attributed to a single element and its digitalization, but rather depend on an interaction effect of digitalization of multiple elements.

Transferring this distinction of local changes versus complex changes to the digital transformation of radio broadcasting, research has thus far primarily been concerned with local changes due to the digitization of, for example, program production or distribution channels [13]. With respect to program production, digital technologies have replaced analogue technologies for storing and accessing music and other audio recordings, post-processing audio recording (e.g., change amplitude or frequency, cut multiple recordings), or mixing multiple lines including telephone calls via digital phone lines [14]. Regarding distribution channels, various digital broadcasting standards have been drafted and implemented by radio stations and electronics manufacturers over the last decades including, for example, "Digital Audio Broadcasting" (DAB) and its successor DAB+ or "Digital Video Broadcasting Terrestrial" (DVB-T) [15]. These rather infrastructural changes have not been of particular interest for IS research. However, with the combination of digital and often IP-based infrastructures, abundant internet availability, changing listener attitudes, smart broadcasting devices of all kinds, and other changes, the radio broadcasting industry is already undergoing a more complex and fundamental digital transformation. There has been little research-and virtually none from the IS discipline-regarding more complex changes in radio broadcasting that are enabled by the combination of, for instance, digital program production, digital distribution including internet infrastructures, and digitally enabled playback devices.

Service innovation has been approached from different conceptual angles [3]. Some scholars view services as immaterial offerings made by organizations in addition to - and relatively analogous to - physical products [16]. Thus, similar to product innovation, service innovation is viewed as being market-driven, that is, by changing demand and/or organizations' pursuit for differentiation in new or existing markets [17], [18]. Other scholars argue, however, that albeit being similar to products as offerings by organizations, innovation of services is different because it is (at least initially) driven internally by organizations' striving to increase efficiency, quality, and effectiveness of existing services by means of new technology [19]. Once in place, firms may explore and leverage capabilities of new technologies to create new service offerings geared towards new customer segments or markets.

A fundamentally different perspective refutes the distinction between products and services because, as is argued, virtually an economic exchange involves both service and physical products [20], [21]. This idea has been taken up among others by the proponents of service-dominant logic [22] who argue that service (not goods) is the fundamental basis of economic exchange [23]. Service is defined as "the application of specialized competences (knowledge and skills) through deeds, processes, and performances for the benefit of another entity or the 
entity itself" [22, p. 2]. If an exchange includes goods, they are mere distribution mechanisms for service, because service (i.e., knowledge and skills) has been applied to produce them. Consequently, with respect to value creation, service-dominant logic does not view value as being embedded in produced goods and being evaluated during their exchange (value in exchange). Service-dominant logic rather assumes that value is determined by the customer during service experience (value in use, or value in context). More specifically, value is co-created either in direct interaction of customer and service provider, or when the customer uses the service provider's knowledge and skills that have been embedded into goods upon production [22], [23]. Thus, one can think of service as the integration of resources. This emphasizes that the value of goods does not result from owning them, but from using the service they provide [24]. Further, since value is co-created in use, companies (or service providers, more generally) cannot produce value, but can only make value propositions [22], [23]. Based on this perspective on service, service innovation can be seen as the "rebundling of diverse resources that create novel resources that are beneficial (i.e., value experiencing) to some actors in a given context" [4, p. 161]. Digital technologies are instrumental in service innovation because they enable the digitalization of ever more resources, thus removing the need to transfer humans or tangible goods and making it easier to rebundle and integrate them in new services [4].

\section{Methodology and Data}

Since there has only been little research on how radio broadcasting can be innovated in times of digital transformation, we decided to use an interpretive research approach [25], [26] and a method that allows the researcher to capture rich information without limiting one's perspective through a specific theoretical lens. We chose to analyze expert practitioners' assessment of digital transformation of radio broadcasting by means of qualitative content analysis (QCA). QCA allows the researcher to describe systematically the meaning of qualitative data. A primary goal of this method is to structure and organize obvious and latent content. In QCA, the researcher assigns successive parts of the research material to categories of a coding frame. The coding frame can be derived deductively from theory, inductively from data, or mixed (e.g., main categories from theory, subcategories from data) [27]. The coding frame is at the heart of the method, especially in an exploratory approach where "the coding frame itself can be the main result" [27, p. 180].
In our analysis, we applied QCA to systematically analyze keynote talks on the digital transformation of radio broadcasting from practitioners in or related to the radio broadcasting industry. Our data were six keynote talks of well-selected international experts from the radio broadcasting industry that we invited to give a talk at a full-day workshop on the topic of "Radio-Innovations: On the way to Interactive Radio". The goal was to develop a rich account of what expert practitioners perceived as the main challenges and possible solutions in fundamentally innovating radio broadcasting through digital technologies. The practitioners were free to prepare their talks and decide what to cover. The workshop was hosted in October 2016 by the Institute for Broadcasting Economics, based in Cologne, Germany. Two talks were given in English and four talks in German. The talks were video-recorded and summed up to a total of 163 minutes. Question-andanswers-session following some of the talks were not included in the analysis. Practitioners were affiliated either with broadcasting stations in the US (one practitioner), the UK (one), and Germany (two), or with start-ups related to radio broadcasting (two). By our selection of international practitioners and the mix of start-ups and traditional broadcasting agencies, we intended to make our selection as unbiased as possible, keeping in mind that the selection of data sources is crucial for QCA. Since speakers were free to choose the topic for their talk, we also tried to minimize the selection bias with respect to topics.

We followed the methodological guidelines as described by Schreier (2014), which comprise the following steps: data preparation, building the coding frame, pilot phase, main analysis, and presenting findings. To prepare the data, we transcribed all six talks and time-coded the presenters' slides.

Because of the exploratory nature of our research question we decided to follow a mixed approach to build the coding frame, that is, deductively derive main categories from the research question and inductively generate subcategories from the transliterated talks. To do so, we segmented all transcripts thematically. Further, we iteratively collected potential subcategories from two of the six transcripts and structured them in the coding frame. This included to reassign some codes as subsubcategories to others, merging codes, and rephrasing codes. We stopped when no further changes to the coding frame were necessary. Segmentation, generation, and revision of the coding frame were conducted independently by two of the co-authors. Intermediate results were compared, and conflicts resolved through discussion. 
Usually, a pilot coding is conducted prior to the main analysis to evaluate the coding frame and the agreement between multiple researchers in applying the coding frame, before the coding frame is fixed and the whole material is coded only once by one of the researchers. However, since we recognized substantial need for discussion between the two coders when building the coding frame, we decided to have all talks coded by two researchers, compare results, and resolve potential conflicts through discussion. Thus, we extended the tasks that are usually only applied to parts of the material in a pilot phase to the whole material in the main analysis. This meant more coding work and discussion, but also more consistent results.

\section{Results}

In this section, we present our findings from applying exploratory QCA to the talks of six practitioners from the radio broadcasting industry on the topic of "Radio-Innovations: On the way to Interactive Radio". According to best practices, the presentation of exploratory QCA primarily consists of a presentation and illustration of the coding frame that resulted from the analysis. In other words, the coding frame, its codes, and their meanings are the findings.

The coding frame has three levels, namely main, sub-, and sub-subcategories. Four main categories have been derived from the research question: (1) challenges for radio broadcasters from the digital transformation, either (a) exogenous or (b) endogenous, (2) specifics of radio that will endure the digital transformation and innovation, and (3) solutions for future, digitally innovated radio. Subcategories and sub-subcategories have been collected inductively from the data (i.e., transcripts of talks) and iteratively assigned to the main categories. The presentation of the findings is structured according to the coding frame (Table 1). Original codes of subcategories and sub-subcategories are printed in bold face.

\subsection{Exogenous challenges for RBAs}

Changing customer expectations. The most important exogenous challenge for radio, as mentioned in the talks, are changing customer expectations. First, a radio is no longer a small, dedicated device in the kitchen, in the bathroom, or in the car. Listeners expect radio to accompany them wherever they go, and to be available anytime, anywhere, and on demand. For example, customers do not want to wait up to half an hour to hear the news. "I just missed the news, so I have to wait" (Practitioner F, from a user's perspective). The challenge will be to understand where and when users will want to hear radio. So far, radio broadcasters did not have to do this, or only to a limited degree. "We're doing the same thing, but what we should be doing is rethinking what is possible" (Practitioner B). Practitioners saw radio as ultimately becoming even more ubiquitous.

The popularity of new smartphone apps, especially music streaming apps like Spotify, has created specific customer expectations regarding audio consumption, and customers continuously, yet subconsciously transfer these expectations to other media, such as radio. This also applies to user experience and usability. Many current radio apps do not provide an appealing user experience. They have an old-fashioned design, a moldy look and feel, or are just not attractive enough to make users use them every day. Media centers need to be redesigned.

Further, practitioners felt that the low usage numbers of podcasts, for example, in Germany (between $7 \%$ and $13 \%$ of all internet users depending on the source), are indicative of suboptimal usability, that is, it is burdensome for customers to manage them across multiple stations and devices. The current expectation regarding radio is also that listeners should not need to take care of anything. But expectations can change from generation to generation and with other kinds of psychographics. Since younger listeners tend to transfer expectations from music streaming apps, they increasingly demand to become their own program directors for radio as well. "They expect to hear what they want to hear, not what the broadcaster has decided to offer them" (Practitioner C). This is supported by findings of the Nieman Lab. State of the art apps for music streaming offer an "explore" or "coming up next" screen that allows users to edit playlists and assemble unique experiences. The outcome of similar editing functions in radio would be a stream that almost feels like traditional linear radio program, but is in fact customized.

Being involved in creating the stream is however not a must. There will always be situations where users do not want to interact but rather just consume what is sent by the broadcaster. "Sometimes I turn on the radio because I don't need to care about the program, I am discharged from being my own program director" (Practitioner C, from a user's perspective). Related to the idea of having the possibility to build a custom stream is the expectation to search and browse for interesting content. 
Practitioners assume that younger customers will expect additional multimedia content, for example, photos when consuming radio on their mobile devices, and will expect to be able to skim across a variety of audios much quicker. However, the content itself develops rapidly. Content presented on, for example, YouTube does not adhere to journalistic standards, but is published with a higher frequency and also a higher immediacy. Furthermore, practitioners have observed that, "if content is produced too perfectly, it becomes inauthentic" (Practitioner A) for younger listeners.

Furthermore, future listeners will expect personalization of the content, as they already experience today in ecommerce, movies and music recommendations. Recommender systems for radio is one of the hottest topics in the field of radio innovation. As a state of the art, recommender systems do not only learn users' preferences from their interactions, but also adapt to context factors such as time of day, day of week, location, speed, movement and the like. "If you thought of surround speakers in your living room, you don't want the same experience as if you're on a busy train with some headphones" (Practitioner B).

Another changing expectation we identified is the opportunity to socially interact with the radio program. Consumers nowadays use social networks like Facebook and Snapchat and they do not want to participate in a new social network for sharing radio content. "They expect the content to be where they already are" (Practitioner E). Missing socially enabled offers are considered as one of the main reasons for stagnating consumption of podcasts.

Emergence of new technologies. New customer expectations are rooted in new technologies. A plethora of new devices provides an existing infrastructure, where the supply has to meet the demand. Radio broadcasters face the challenge to meet their listeners on those devices, not the other way around, as it was the case for traditional radio (i.e., listeners bought devices to consume the program). Radio has been an alongside medium ever since. But with the multitude of different devices, different expectations emerge, as stated above. Broadcasters need to be present on all devices because users expect them to be.

A typical application for radio is in cars. Still today, in-car radio has to be designed in a way that ensures a "safe driving experience, but allowing the driver a lot more of advanced controls, with his eyes on the road" (Practitioner A). But there is already a second challenge coming up with self-driving cars. In self-driving cars, entertainment in the car can widely open up, and radio will face increased competition when people become interested in visual content rather than audio content.

Compared to all those new devices that emerge on the listener side, that are outdated every two or three years, and that constantly need hardware updates and software updates to provide new functionalities, the speed of technological development in the radio broadcasting industry is disproportionately slow.

Changing competition landscape. Practitioners saw the radio broadcasting industry to be in a digital paradigm shift that is similar to what happened in the music industry and the film industry 15 years ago. While music and film have been changing and specific new offerings have been innovated, radio broadcasting still has to work out how this paradigm shift will look like for them. Currently, many radio broadcasters are not able to develop digital products such as apps and at the same time make sure that appropriate new content formats are delivered. "Software development is impossible for a lot of broadcasters today" (Practitioner D). Some practitioners questioned whether broadcasters can pursue a digital product strategy without having inhouse digital development competencies, but rather contracting with service providers. While radio broadcasters had their own territory for some decades, nowadays they find themselves in a situation of increasing competition with other media companies due to an ongoing convergence of media. This results in a threat from digital media companies. Radio broadcasters have to compete with big players like Apple Music, Spotify, and Google, that may prescribe in which ways radio content shall be delivered through their platforms. Furthermore, big players keep updating their products and continuously invest a lot of money to keep their platforms going. Change processes on those platforms take place in short cycles of months or years, and they are multidimensional. Hence, radio broadcasters have to face the challenge that their service portfolio may fall back behind all other media consumption possibilities. RBAs have to question themselves how they can keep listeners engaged, that is, how they can get them to consume another piece, or listen for another minute.

The radio audience is aging [28]. The fear to lose younger customer segments is a direct consequence, also called the generation tear-off. New media companies compete for younger customer segments, and radio broadcasters have "already lost relevance in certain user segments and in certain age groups" (Practitioner D). Radio broadcasters face the challenge of approaching a younger audience, and 
ask themselves the questions: "How do we expand the reach of people interacting with our content or our brand? How are we going out and finding new consumers? What effort do we have in that regards?" (Practitioner A).

Compliance with Public-Service Remits. When losing customer segments, another exogenous challenge comes into play for publicly financed RBAs: the compliance with public-service remits. The service offer should support the process of forming a free and individual opinion and therefore fulfil the needs of a democratic, social and cultural society. "The goal for publicly financed broadcasters is not necessarily to increase retention time of consumers on digital products or to show them as many as advertisements as possible" (Practitioner D). Normative goals are defined by exogenous broadcasting acts. Therefore, a special challenge arises for the design of applications, especially recommender systems and personalization.

\subsection{Endogenous challenges for RBAs}

Apart from exogenous challenges, some (but fewer) endogenous challenges have been mentioned. A first endogenous challenge is the convergence of media. Journalists have been categorized according to a specific medium (i.e., newspaper, TV, radio) for a long time, and many of them still think in terms of radio journalists, TV journalists, and newspaper journalists. This is also still the case for the training of journalists, although the justification for a distinction according to the medium diminishes.

A second endogenous challenge is that radio broadcasters had undergone a long period of little to no change of their business model, and now need to catch up in a fast-changing environment. "The times, in which the technical conditions were set for decades, are virtually over, and will never come back" (Practitioner D). Existing infrastructure of RBAs has specific production processes that are optimized for linear mass distribution. The existing infrastructure lacks flexibility to support modern ways of media distribution.

Broadcasters have huge amounts of high-quality content, but most content is played-out only once or twice. Linear radio is designed in a way, that, after it has been broadcasted, it is gone. Tape archives exist for content that has already been on air, but it is difficult to retrieve specific recordings from these archives and broadcasting slots are required to send it again. Practitioners perceived it as rather unsatisfying to own loads of costly-produced content without having appropriate possibilities to make it available to the public.

A last endogenous challenge is the limited availability of metadata. Historically, radio had no screen and metadata was not needed. "We linear radio people do not have additional metadata, apart from the metadata that we traditionally know from car radio" (Practitioner C). The invention of Radio Data Stream (RDS) did also merely change that. Metadata are typically created by archivists after content has been broadcasted and only for the purpose of archiving. However, metadata are necessary for content-based recommendation techniques and should therefore be created and made available during production of radio programs. Current infrastructures, organizational structures, and processes do, however, not support metadata.

\subsection{Specific characteristics of radio}

In this subsection, we summarize what practitioners identified to be specific characteristics of radio that differentiate it from other media.

A radio program is traditionally structured according to the radio hour clock. Every radio show of, for instance, 30 or 60 minutes includes several different types of audio content (e.g., music, spoken, news). The radio clock divides the total length of the show into smaller parts and defines which type of content is played at which time. Different radio hour clocks are used for different radio programs. This tool provides some sort of orientation to both listeners and editors and also shapes the specific contemporary radio experience. When creating a nonlinear, customized radio program, RBA need to think about whether to abandon this tool at all or how to transfer and modify it. If it should be transferred, it might require additional work for cutting long audios into smaller parts that can be used in different radio playouts. It also requires rethinking the sequencing and embedding sequence logic into algorithms.

Radio has a strong market segmentation according to languages. Furthermore, a lot of countries have both publicly and privately financed broadcasting agencies. As both types follow different goals, a public-private divide exists, which makes it more difficult to create joint offers with respect to personalization.

Radio comprises some specific aesthetics. Since radio receivers had become smaller and transportable, radio has increased its importance as an alongside medium over the years. This means that people usually consume radio while doing something else besides, such as getting prepared in the bathroom, driving a car, or washing up dishes. The 
characteristics of the alongside medium is also closely linked to habits and daily routines, and the characteristic of radio as being simple to use. Possible interactions are reduced to a handful of switches or buttons: turn on, turn off, volume, change channel. Further, radio broadcasts a mixture of formats like stories, articles, interviews, long features, and audio dramas. The heterogeneity of formats gives radio a diversified image.

Immediacy is another important characteristic of radio. Radio often manages to evoke the impression of being live, even when it is not. The image of the moderator talking to all listeners at the same time might be difficult to keep in a nonlinear program.

\subsection{Solutions for future radio}

Proposed solutions how to design future radio are manifold. They range from visions of new digital products and services to concepts of organizational and technical change.

Follow an audience strategy. According to the practitioners, besides all other innovative ideas, RBAs should strictly pursue an audience strategy and put the audience first. The audience strategy should identify new audience segments and define dedicated target levels of reach. It should consider the way RBAs are going to engage with listener segments in the world they live in. RBAs should measure actual reach ranges and thus decide whether if existing offerings should be kept, changed, or turned off. Everything RBAs do should be about their relationship with the audience. The strategy should ensure that RBAs are not building something for anything, but rather enable them to focus on who they are trying to reach. Especially younger audiences will be addressed by an audience first strategy, and continuous investigations on trends on the internet are required. In this regard, the digital strategy for RBAs is primarily an audience first strategy.

Cultivate consumer interaction. Practitioners propose to think of consumer interaction as a lifecycle of three elements: reach, engagement, and connection. Once RBAs managed to reach their listeners, they should try to get them engaged, and ideally establish a connection, that is, become a habit in people's life. The listeners that enter into a connection with the brand and the offering act as evangelists who reach out to new customers. The central requirement is that RBAs manage "to meet or exceed listeners' expectations" (Practitioner A). They can do so by learning from feedback data, not omitting it, for differentiated target groups. The feedback channel needs to receive increased attention because feedback data represent facts about listeners' evaluation of the offering, rather than opinions. In case feedback data does not support opinions and positions on innovation and both stand in conflict, data should trump opinions, even if this is not pleasant. RBAs should link their content to social media, not just to meet customers there and leverage their content in new ways, but also because social media supports rich feedback and exchange possibilities. With an audience first strategy, RBAs can manage to integrate their content and their brand into listeners' daily routines and "to be one of the three apps they use on a daily basis" (Practitioner A).

Follow a joint content and digital service strategy. RBAs should not follow a content strategy alone as they did in the last decades. Next to the requirement to provide high quality content, they have to make sure the content is being heard at the place where they want to be heard. Therefore, they have to design their digital offerings accordingly, and both have to go hand-in-hand. The plethora of devices induces that there is a stronger tie between content and device than before, and so content and distribution of contents have to follow a joint strategy.

Build up in-house development competences. In order to become a creative lab for digital radio, RBAs have to build up development competences for digital services on their own. "I believe we also have to become software developing companies, otherwise this won't work" (Practitioner D). Digital products and services should not be developed outside of the RBA, because these products exist in a quickly changing environment. RBAs do not have to build one digital product or service, they have to build several, and it is not a one-time effort, but ongoing. Constant adaption and renewing of digital offerings is necessary. Agile development methods and lean startup methods seem appropriate for RBA digital development teams, as they can produce new services and products as quickly as possible, put them in front of real people, and measure how they respond to it.

New governance. RBAs must bring themselves into a position to be a digital company, to "develop digital products and services jointly with the program" (Practitioner D) and make them ready for the market. This requires changes in governance and processes. In current RBAs, technology is often regarded as a service provider to content. In the future, this needs to change, because developers have specific perspectives and knowledge on how digital products and services should look like, and journalists and editors have specific competences in content creation. Both need to work together on an equal footing to create successful products and 
services. Practitioners argued that this should also be reflected in organizational structures, for example, by include technology at the C-level. RBAs should establish a digital board, which develops the goals and the "big picture" for the RBA's digital initiatives. All initiatives and ideas that are brought up by either technology or content are evaluated by the digital board and checked for strategic alignment. Thus, RBAs can coordinate digital measures. Software development processes should be adapted to the perspectives and processes of journalists and editors. Further, new job roles can be established such as an innovation accountant, who should encourage people to state hypotheses about what is going to work and provides feedback metrics to understand which features are working well and which people are not engaging with.

Open up to new communities. RBAs should open up to new developer communities by providing their content via API on several service layers and to provide it in a developer center. Once digital products and services draw attention, a lot of prospective partners are encouraged to build apps on their own. Therefore, RBAs should consider "building frameworks rather than platforms" (Practitioner A). Thus, RBAs can save development resources and at the same time gain reach quicker. Further, research cooperations gain importance. Radio still poses a challenge for recommender systems. For building digital products and services, RBAs need young talents with high affinity to media, motivated designers and developers, to establish some start-up atmosphere. Recruiting should be designed accordingly, with hackathons or meetups.

Leverage content in new ways. First, social media integration has the power to leverage content. New technologies like jump marks to a specific place in an audio can effectively support this. A second promising way is the use of recommender systems. RBAs should provide services to consume their content personalized. A mix of editorial content curation and collaborative filtering has turned out to be even better than collaborative filtering alone. The power of RBAs can therefore lie in the combination of personalization, which is new to RBAs and where they can improve on over time, with content curation competences, their established experience field.

Change internal processes to generate metadata earlier. In contrast to linear distribution structures in which metadata is typically generated at the end for archiving purposes, RBAs should consider changing processes and "generate metadata already in production" (Practitioner C), as metadata becomes more and more important for digital products and services in nonlinear distribution.
Table 1. Coding frame

\begin{tabular}{|c|c|}
\hline $\begin{array}{l}\text { Main } \\
\text { category }\end{array}$ & Sub- and sub-subcategories \\
\hline $\begin{array}{l}\text { 1a) } \\
\text { Exogenous } \\
\text { challenges } \\
\text { for RBAs }\end{array}$ & $\begin{array}{l}\text { Changing customer expectations } \\
\text { ubiquitous; user experience; } \\
\text { usability; own program } \\
\text { directors; search and browse for } \\
\text { interesting content; } \\
\text { recommender systems } \\
\text { Emergence of new technologies } \\
\text { plethora of new devices } \\
\text { Changing competition landscape } \\
\text { threat from digital media } \\
\text { companies; generation tear-off } \\
\text { Compliance with public-service remit }\end{array}$ \\
\hline $\begin{array}{l}\text { 1b) } \\
\text { Endo- } \\
\text { genous } \\
\text { challenges } \\
\text { for RBAs }\end{array}$ & $\begin{array}{l}\text { convergence of media; long period of } \\
\text { little to no change; optimized for } \\
\text { linear mass distribution; huge } \\
\text { amounts of high-quality content } \\
\text { played out only once or twice; limited } \\
\text { availability of metadata }\end{array}$ \\
\hline $\begin{array}{l}\text { 2) } \\
\text { Specific } \\
\text { charac- } \\
\text { teristics } \\
\text { of radio }\end{array}$ & $\begin{array}{l}\text { radio hour clock; market } \\
\text { segmentation according to languages; } \\
\text { public-private divide; specific } \\
\text { aesthetics; alongside medium; simple; } \\
\text { diversified image; immediacy; live; } \\
\text { surprising moments }\end{array}$ \\
\hline $\begin{array}{l}\text { 3) } \\
\text { Solutions } \\
\text { for future } \\
\text { radio }\end{array}$ & $\begin{array}{l}\text { Follow an audience strategy } \\
\text { Cultivate consumer interaction } \\
\text { a habit in people's life; learning } \\
\text { from feedback data; link content } \\
\text { to social media } \\
\text { Follow a joint content and digital } \\
\quad \text { service strategy } \\
\text { Build up in-house development } \\
\quad \text { competences } \\
\text { New Governance } \\
\text { Open up to new communities } \\
\text { building frameworks rather than } \\
\text { platforms } \\
\text { Leverage content in new ways } \\
\text { Generate metadata earlier }\end{array}$ \\
\hline
\end{tabular}

\section{Discussion and conceptualization from the perspective of service-dominant logic}

A wide array of challenges, solutions, and specific characteristics of radio broadcasting have been mentioned in the talks. In this section, we reconceptualize the various aspects of digital transformation of radio broadcasting from the 
perspective of service innovation as conceptualized according to service-dominant logic [4].

Lusch and Nambisan [4] propose to structure service innovation into three domains, namely, service ecosystem, service platform, and value cocreation. A service ecosystem is a "relatively selfcontained, self-adjusting system of mostly loosely coupled social and economic (resource-integrating) actors connected by shared institutional logics and mutual value creation through service exchange" [4, p. 162]. The service platform within a service ecosystem is a "modular structure that consists of tangible and intangible components (resources) and facilitates the interaction of actors and resources (or resource bundles)" [4, p. 162]. Value co-creation refers to the "processes and activities that underlie resource integration and incorporate different actor roles in the service ecosystem" [4, p. 162]. Lusch and Nambisan [4] highlight key issues that organizations need to address for service innovation with respect to these three domains.

Regarding value creation, key roles and the nature of value (co)creation need to be defined, and a supportive environment for resource integration needs to be created, that is, mechanisms to enable interaction between diverse actors, suitable internal processes, and transparency of resource integration. Applied to radio service innovation, this means that broadcasters need to think strategically about the role of listeners and other resource-providing actors (e.g., podcasters, app developers) for value co-creation within their service ecosystem. So far, radio broadcasters do realize the new (inter-)active role of listeners and they also reassess their diverse internal resources (e.g., knowledge and skills of editors, program managers). They realize the need to facilitate the creation of new digital services, yet only few practitioners are considering to open up for and to cooperate with new actors to do so. Moreover, little have we found about how radio broadcasters plan to establish supportive environments that attract actors with particular roles and resources to their service ecosystem.

Key issues with respect to the service ecosystem are to maintain its structural flexibility and integrity, to facilitate a shared worldview among actors, and to provide an architecture platform for service exchanges [4]. Similar to the questions which new actors to attract and how, broadcasters are not yet considering issues of structural flexibility (e.g., how to allow new actors in the radio service ecosystem to adapt to new listener preferences?) and integrity (e.g., ensure some degree of continuity in cocreation of new radio services, despite the flexibility of individual actors). On the other hand, broadcasters in our sample did display a particular set of characteristics that they view as distinctive for radio which could serve as basis for a shared worldview for new radio services. However, broadcasters would also need to share this view with other actors in their ecosystems and to develop it further. Lastly, the architecture platforms that broadcasters are building for new radio services lack clear concepts for the actual implementation of interaction with listeners as well as resource integration with new actors. For example, it is still unclear to what degree new radio services will rely on explicit (e.g., pushing buttons) or implicit (e.g., skipping tracks) listener feedback, and how new content providers will be able to add their content to the service as well as receive feedback and compensation. This is not merely a question of user interface design, but also affects, for example, the types and implementation of recommender systems that are supposed to integrate particular content with specific listeners.

Key issues of service innovation in the domain of the service platform are to develop an appropriate modular architecture to improve resource density and to establish protocols for exchange of services [4]. Although radio broadcasters are building up internal competencies that enable them to implement suitable service platforms, the impression was that the need to integrate new actors in a modular architecture and to provide suitable protocols has not yet been fully realized by radio broadcasters.

\section{Conclusion, limitations, and future research}

Radio broadcasting, like other media before, is undergoing a fundamental digital transformation. Our work consolidates the perspectives of practitioners on specific challenges and possible solutions how to innovate what can be seen as the service offering of radio, while preserving its specific aesthetic characteristics. Hence, we contribute to the understanding of the phenomenon and provide a theoretical structuration from the perspective of service-dominant logic.

Further, our findings can provide orientation to practitioners in RBAs regarding how to react to challenges of digital transformation. Lastly, our work establishes a fruitful area for IS research. As radio becomes a digital service, knowledge about how to design successful digital user experiences will become important and findings from IS literature can be especially helpful.

Certainly, our work is not without limitations. Since specific solutions for digitally transformed radio are manifold, the solutions we extracted from 
the expert talks are presumably not exhaustive. Especially the area of creating innovative products and services, such as, the possibility to individually vary the length of the pieces, to vary the trade-off between background noise and speaker's voice in live games, or immersive audio, as advanced by the BBC, provide enormous creative potential to provide appealing listening experiences.

Further, while talks from practitioners were very insightful and rich, more stakeholders from the radio broadcasting industry should be included in future research on this topic.

\section{References}

[1] Spotify, "Number of Spotify monthly active users worldwide from 2015 to 2017," 2018. [Online]. Available:

https://www.statista.com/statistics/367739/spotifyglobal-mau/. [Accessed: 08-Aug-2018].

[2] TechCrunch, "Number of paying Spotify subscribers worldwide from July 2010 to January 2018," 2018. [Online]. Available: https://www.statista.com/statistics/244995/numberof-paying-spotify-subscribers/. [Accessed: 08-Aug2018].

[3] M. Barrett, E. Davidson, J. Prabhu, and S. L. Vargo, "Service Innovation in the Digital Age: Key Contributions and Future Directions," MIS Quarterly, vol. 39, no. 1, pp. 135-154, Mar. 2015.

[4] R. F. Lusch and S. Nambisan, "Service Innovation: A Service-Dominant Logic Perspective," MIS Quarterly, vol. 39, no. 1, pp. 155-176, Mar. 2015.

[5] A. Andal-Ancion, P. A. Cartwright, and G. S. Yip, "The Digital Transformation of Traditional Businesses," MIT Sloan Management Review, vol. 44, no. 4, pp. 34-41, Summer 2003.

[6] M. Fitzgerald, N. Kruschwitz, D. Bonnet, and M. Welch, "Embracing Digital Technology," MIT Sloan Management Review (Research Report), Nov. 2013.

[7] G. Westerman, D. Bonnet, and A. McAfee, "The Nine Elements of Digital Transformation," MIT Sloan Management Review (Opinion \& Analysis), Jan. 2014.

[8] A. Majchrzak, M. L. Markus, and J. Wareham, "Designing for Digital Transformation: Lessons for Information Systems Research from the Study of ICT and Societal Challenges," MIS Quarterly, vol. 40, no. 2, pp. 267-278, Jun. 2016.

[9] A. Bharadwaj, E. Sawy, O. A, P. A. Pavlou, and N. Venkatraman, "Digital Business Strategy: Toward a Next Generation of Insights," MIS Quarterly, vol. 37, no. 2, pp. 471-482, 2013.

[10] P. L. Drnevich and D. C. Croson, "Information Technology and Business-Level Strategy: Toward an Integrated Theoretical Perspective," MIS Quarterly, vol. 37, no. 2, pp. 483-509, Jun. 2013.

[11] R. Agarwal, G. Gao, C. DesRoches, and A. K. Jha, "Research Commentary-The Digital
Transformation of Healthcare: Current Status and the Road Ahead," Information Systems Research, vol. 21, no. 4, pp. 796-809, Dec. 2010.

[12] A. Susarla, J.-H. Oh, and Y. Tan, "Social Networks and the Diffusion of User-Generated Content: Evidence from YouTube," Information Systems Research, vol. 23, no. 1, pp. 23-41, Apr. 2011.

[13] A. Dubber, Radio in the Digital Age. John Wiley \& Sons, 2013.

[14] M. C. Keith, The Radio Station, 8th ed. Focal Press, 2012.

[15] B. O'Neill, "DAB Eureka-147: A European vision for digital radio," New Media Society, vol. 11, no. 1-2, pp. 261-278, Jan. 2009.

[16] I. Miles, Services Innovation: A Reconfiguration of Innovation Studies. PREST, University of Manchester, 2001.

[17] F. Damanpour, R. M. Walker, and C. N. Avellaneda, "Combinative Effects of Innovation Types and Organizational Performance: A Longitudinal Study of Service Organizations," Journal of Management Studies, vol. 46, no. 4, pp. 650-675, Jun. 2009.

[18] J. Matthews and A. D. Shulman, "Competitive advantage in public-sector organizations: explaining the public good/sustainable competitive advantage paradox," Journal of Business Research, vol. 58, no. 2, pp. 232-240, Feb. 2005.

[19] R. Barras, "Towards a theory of innovation in services," Research Policy, vol. 15, no. 4, pp. 161173, Aug. 1986.

[20] J. Bryson, P. Daniels, B. Warf, P. Daniels, and B. Warf, Service Worlds: People, Organisations, Technologies. Routledge, 2013.

[21] A. von Nordenflycht, "What is a Professional Service Firm? Towards a Theory and Taxonomy of Knowledge Intensive Firms," Social Science Research Network, Rochester, NY, SSRN Scholarly Paper ID 1407347, Jun. 2010.

[22] S. L. Vargo and R. F. Lusch, "Evolving to a New Dominant Logic for Marketing," Journal of Marketing, vol. 68, no. 1, pp. 1-17, Jan. 2004.

[23] S. L. Vargo and R. F. Lusch, "Service-dominant logic: continuing the evolution," $J$. of the Acad. Mark. Sci., vol. 36, no. 1, pp. 1-10, Mar. 2008.

[24] P. Kotler, Marketing Management: Analysis, Planning, Implementation, and Control, 3rd ed. Upper Saddle River, NJ: Prentice Hall, 1977.

[25] M. D. Myers and H. K. Klein, "A Set of Principles for Conducting Critical Research in Information Systems," MIS Q., vol. 35, no. 1, pp. 17-36, Mar. 2011.

[26] G. Walsham, "Doing interpretive research," European Journal of Information Systems, vol. 15, no. 3, pp. 320-330, Jun. 2006.

[27] M. Schreier, "Qualitative Content Analysis," in The SAGE Handbook of Qualitative Data Analysis, U. Flick, Ed. London, UK: SAGE Publications Ltd, 2014, pp. 170-183.

[28] P. Macavock, "We (still) love radio!," tech-i, no. 31, Mar. 2017. 